\title{
Improved synthesis of 2-arylhydrazono-3-hydroxy-1-propanones and their utility in efficient synthesis of pyridazine derivatives
}

\author{
Nouria A. Al-Awadi, ${ }^{* a}$ Maher R. Ibrahim, ${ }^{a}$ Alya M. Al-Etaibi, ${ }^{b}$ and Mohamed H. Elnagdi ${ }^{\mathrm{a}}$ \\ ${ }^{a}$ Chemistry Department, Faculty of Science, Kuwait University, P.O. Box 5969, \\ Safat 13060, Kuwait \\ ${ }^{b}$ Natural Science Department, College of Health Sciences, Public Authority for Applied \\ Education and Training, Kuwait \\ E-mail: n.alawadi@ku.edu.kw
}

DOI: $\underline{\text { http://dx.doi.org/10.3998/ark.5550190.0012.225 }}$

\begin{abstract}
Reaction of 2-arylhydrazono-1-phenylethanones 2a-c with formaldehyde either in presence of montmorillonite K10 or by simply stirring in methanol and TEA leads to selective synthesis of 2arylhydrazono-3-hydroxy-1-phenylpropan-1-one derivatives 3a-c in high yield. Heating 1a with $N$-phenylmaleimide in DPE/DABCO using microwave irradiation for 5 minutes at $200{ }^{\circ} \mathrm{C}$ results in a $73 \%$ yield of pyrrolo[3,4-c]pyridazine 8. Compounds 2a-c react with benzylidenemalononitriles $9 \mathbf{a}$ or ethyl 2-cyanocinnamate $\mathbf{9 b}$ in refluxing ethanol and piperidine or $\beta$-chitosan to yield new pyridazine derivatives $\mathbf{1 0 a - f}$ which are converted into pyridazinones 11a-c upon reflux in acetic/hydrochloric acids mixture.
\end{abstract}

Keywords: Arylhydrazonals, pyridazines, pyrrolopyridazine

\section{Introduction}

Pyridazines and their derivatives although known for more than a century ${ }^{1}$ received little attention until the discovery of medicinally useful natural products. ${ }^{2-4}$ Today, the pyridazine nucleus has been recognised as a versatile pharmacophore. This key structure is a constituent in many biologically active substances. ${ }^{5-12}$ Recently we reviewed synthetic approaches to pyridazines and condensed pyridazines. ${ }^{13}$ We have previously reported the synthesis of arylhydrazonals 1a-c by heating of $\alpha$-methyl-ketone with dimethylformamide dimethyl acetal (DMFDMA) for five hours in xylene and coupling the resulting enaminones with an aromatic diazonium chloride (Scheme 1). ${ }^{14}$ 


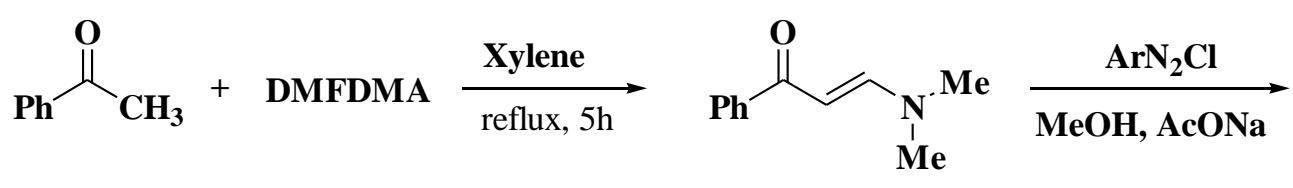<smiles>O=CC(=NNC(=O)c1ccccc1)C(=O)c1ccccc1</smiles>

1a-c

\section{Scheme 1}

In the present work we report an alternative approach for the synthesis of arylhydrazonals 1a-c in good yield from 2-arylhydrazono-1-phenylethanone derivatives 2a-c (Scheme 2). Arylhydrazonal 1a was converted into a pyridazine via a Baylis-Hillman reaction with methyl vinyl ketone; we interpreted this ${ }^{15}$ as involving the ready transformation of intermediate $\mathbf{4}$ into $\mathbf{5}$ which, on loss of water, yielded pyridazine $\mathbf{6}$. In this work, the intermediate $\mathbf{5}$ has been isolated and fully characterized. Compound 2a was heated with $N$-phenylmaleimide to form a new pyrrolo[3,4-c]pyridazine 8. Compounds 2a-c were reacted with $\mathbf{9 a , b}$ to produce new pyridazine derivatives 10a-c, 11a-c.

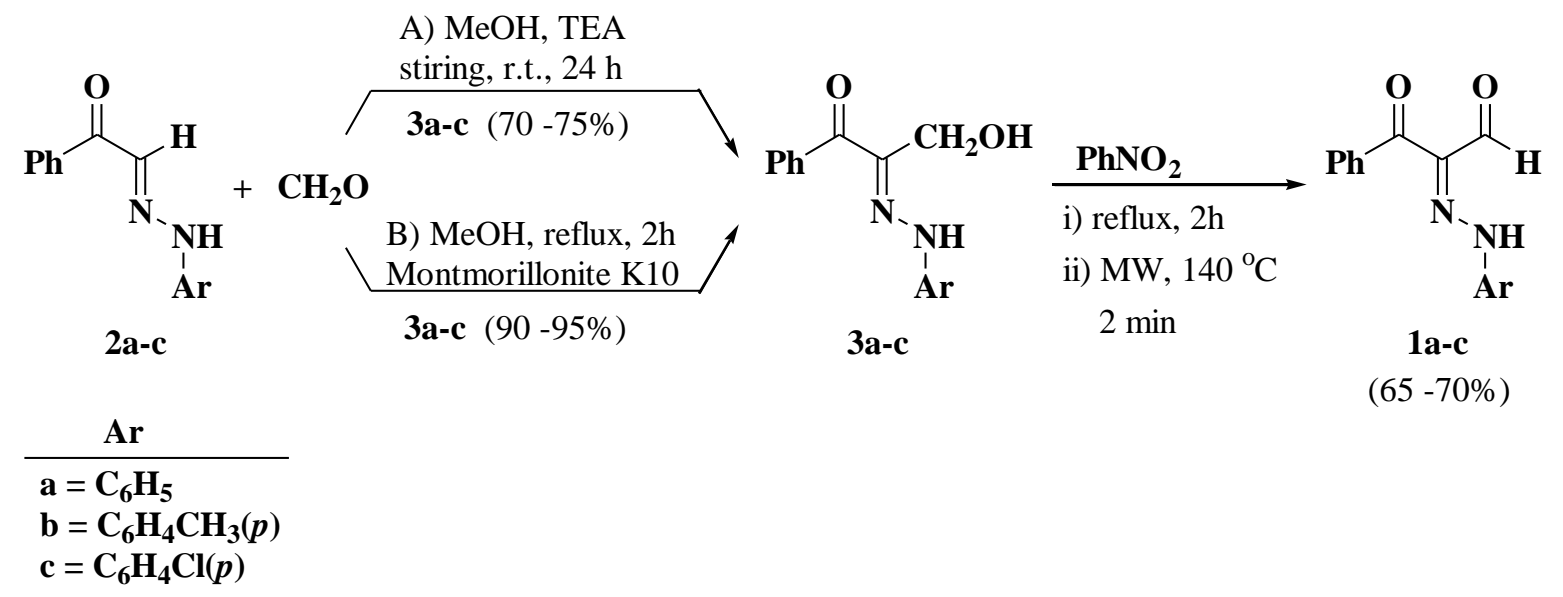

\section{Scheme 2}

\section{Results and Discussion}

Scheme 2 illustrates the selective synthesis of 2-arylhydrazono-3-hydroxy-1-phenylpropan-1ones 3a-c by reaction of arylhydrazono-1-phenylethanones 2a-c with formaldehyde, using montmorillonite $\mathrm{K} 10$ as a shape selective catalyst that favours formation of slim molecules, by reflux in methanol or by simply stirring 2a-c in methanol in presence of triethylamine with formaldehyde, yielding 3a-c in 90-95\% and 70-75\% yields respectively. Refluxing 3a-c with 
nitrobenzene as solvent and oxidizing agent resulted in the formation of arylhydrazonals 1a-c in $65-70 \%$ yields.

We have previously reported the reaction of 1a with methyl vinyl ketone using microwave heating in the presence of DABCO to prepare 1-(6-benzoyl-2-phenyl-2,3-dihydropyridazine-4yl)ethanone $\mathbf{6}$ in $92 \%$ yield. ${ }^{15}$ This reaction was assumed to proceed via intermediates $\mathbf{4}$ and $\mathbf{5}$. In the present work we have successfully isolated 5 in $78 \%$ yield and converted it into $\mathbf{6}$ via microwave heating for five minutes at $160{ }^{\circ} \mathrm{C}$ in $93 \%$ yield (Scheme 3 ).

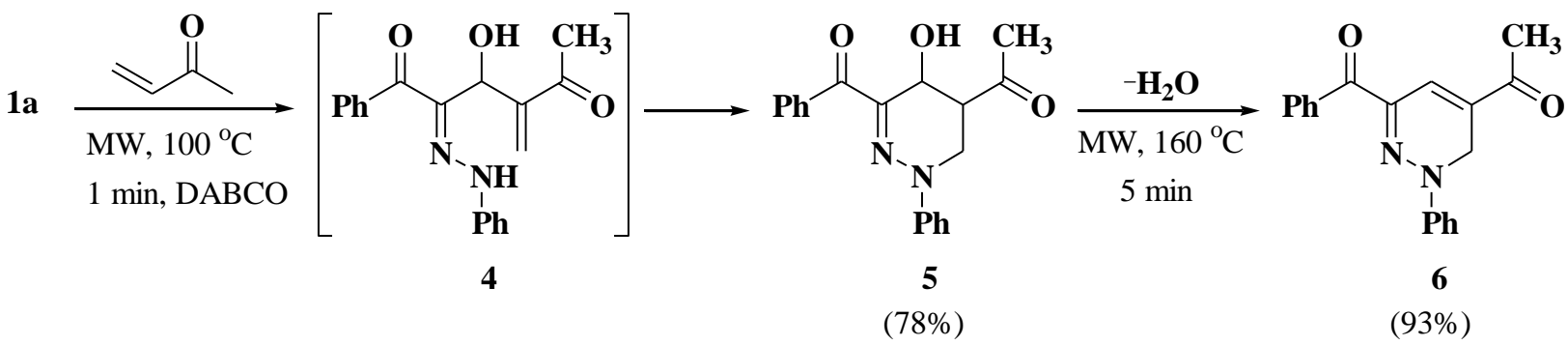

\section{Scheme 3}

The structure of compound 5 was confirmed by 2D NMR experiments. Figure 1 shows ${ }^{1} \mathrm{H}$ and ${ }^{13} \mathrm{C}$ NMR signal assignments and the H-C correlations in the HMBC 2-D experiment: $\mathrm{H}-6$ at $\delta: 3.74,4.23$ correlates with C-5, C-12 at 46.8, 204.7; H-5 at $\delta: 2.88$ correlates with C-6, C-12 at 39.6, 204.7; $\mathrm{H}-4$ at $\delta: 5.36$ correlates with $\mathrm{C}-3$ at $139.5 ; \mathrm{H}-9$ at $\delta: 8.00$ correlates with $\mathrm{C}-7, \mathrm{C}-11$ at $191.5,131.9 ; \mathrm{H}-10$ at $\delta: 7.48$ correlates with $\mathrm{C}-8$ at $137.2 ; \mathrm{H}-11$ at $\delta: 7.56$ correlates with $\mathrm{C}-9$ at $130.5 ; \mathrm{H}-13$ at $\delta: 2.45$ correlates with $\mathrm{C}-5, \mathrm{C}-12$ at $46.8,204.7 ; \mathrm{H}-15$ at $\delta: 7.37$ correlates with $\mathrm{C}-17$ at $123.8 ; \mathrm{H}-16$ at $\delta: 7.37$ correlates with $\mathrm{C}-14$ at $145.6 ; \mathrm{H}-17$ at $\delta: 7.11$ correlates with $\mathrm{C}-15$ at 116.1 .
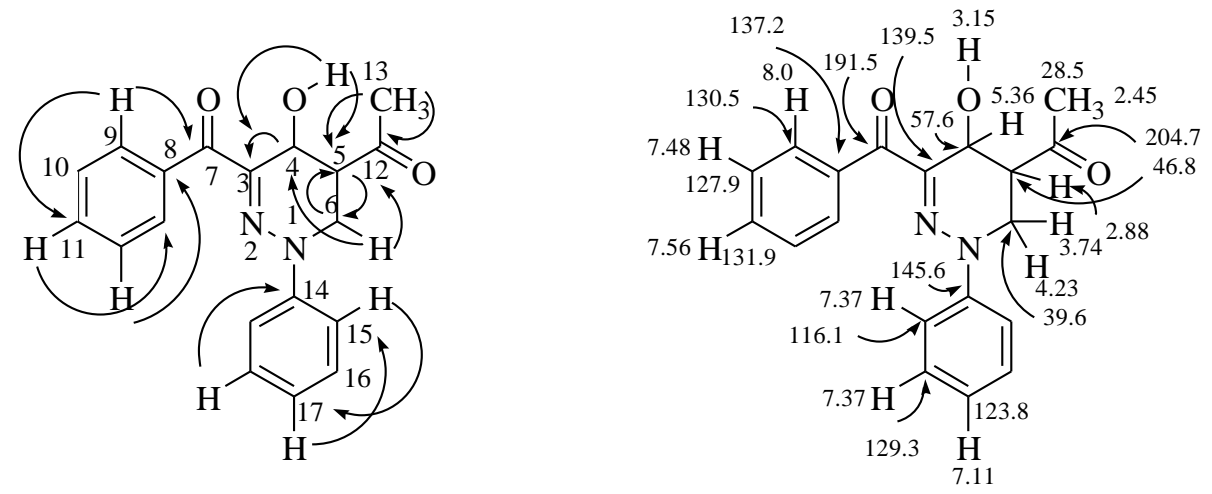

Figure 1. Important HMBC H-C correlation of compound 5.

The X-ray structure of compound $\mathbf{6}$ confirms its structure as that shown in Figure 2. 


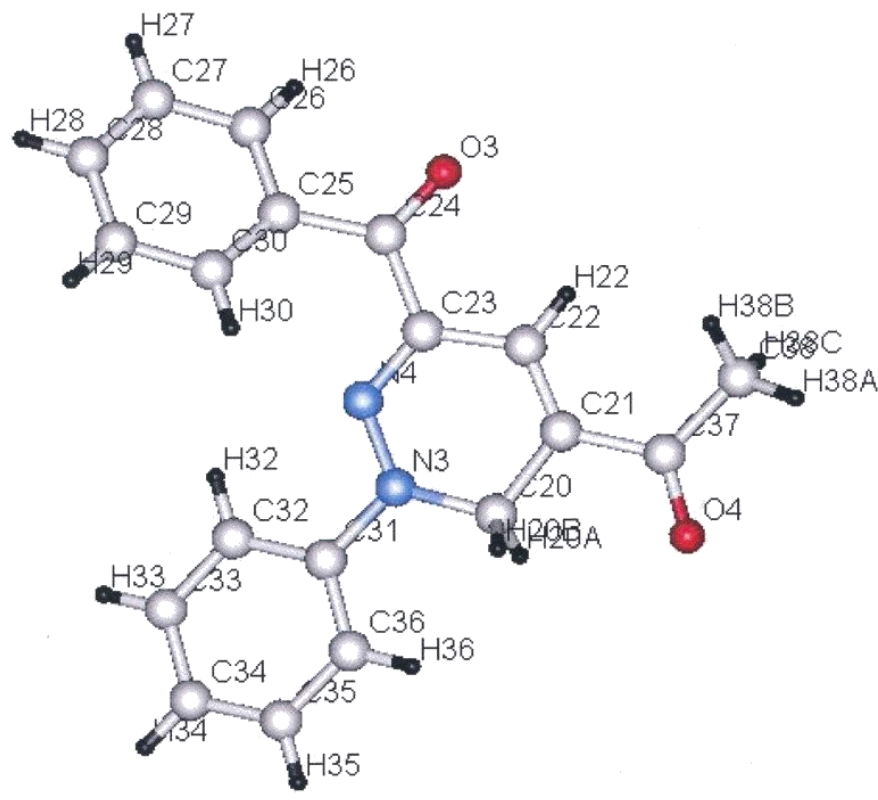

Figure 2. X-Ray crystallographic analysis of compound 6 (ball and stick representation)

Microwave heating of $\mathbf{1 a}$ with $N$-phenylmaleimide at $200{ }^{\circ} \mathrm{C}$ for five minutes in diphenyl ether and DABCO gave 3-benzoyl-1,6-diphenyl-1,2-dihydropyrrolo[3,4-c]pyridazine-5,7-dione 8 in $73 \%$ yield via presumed intermediate 7 (Scheme 4).<smiles>O=C1C=CC(=O)N1c1ccccc1</smiles><smiles>Cc1ccccc1</smiles><smiles>[CH2]O</smiles><smiles></smiles><smiles>O=C(C1=CC2=C(C(=O)N(c3ccccc3)C2=O)N(c2ccccc2)NC1c1ccccc1)c1ccccc1</smiles>

\section{Scheme 4}

Refluxing 2a-c with benzylidenemalononitriles 9a or ethyl 2-cyanocinnamate $\mathbf{9 b}$ in ethanol in the presence of piperidine gave the corresponding 2,5-dihydropyridazine derivatives 10a-f in 
good yields. Compounds 10a-f were also obtained when 2a-c and 9a,b were irradiated in the microwave oven at $160{ }^{\circ} \mathrm{C}$ for five minutes. We have recently reported that $\beta$-chitosan is an efficient basic catalyst for a variety of Michael additions. ${ }^{16 a}$ Replacing piperidine with $\beta$ chitosan afforded 10a-f in almost the same yields. Compounds 10a-c were converted into the corresponding 2,3-dihydropyridazinones 11a-c upon refluxing for four hours in acetic/hydrochloric acids mixture (Scheme 5). Hydrolysis of the amino function under these conditions is well established. ${ }^{16 \mathrm{~b}, \mathrm{c}}$

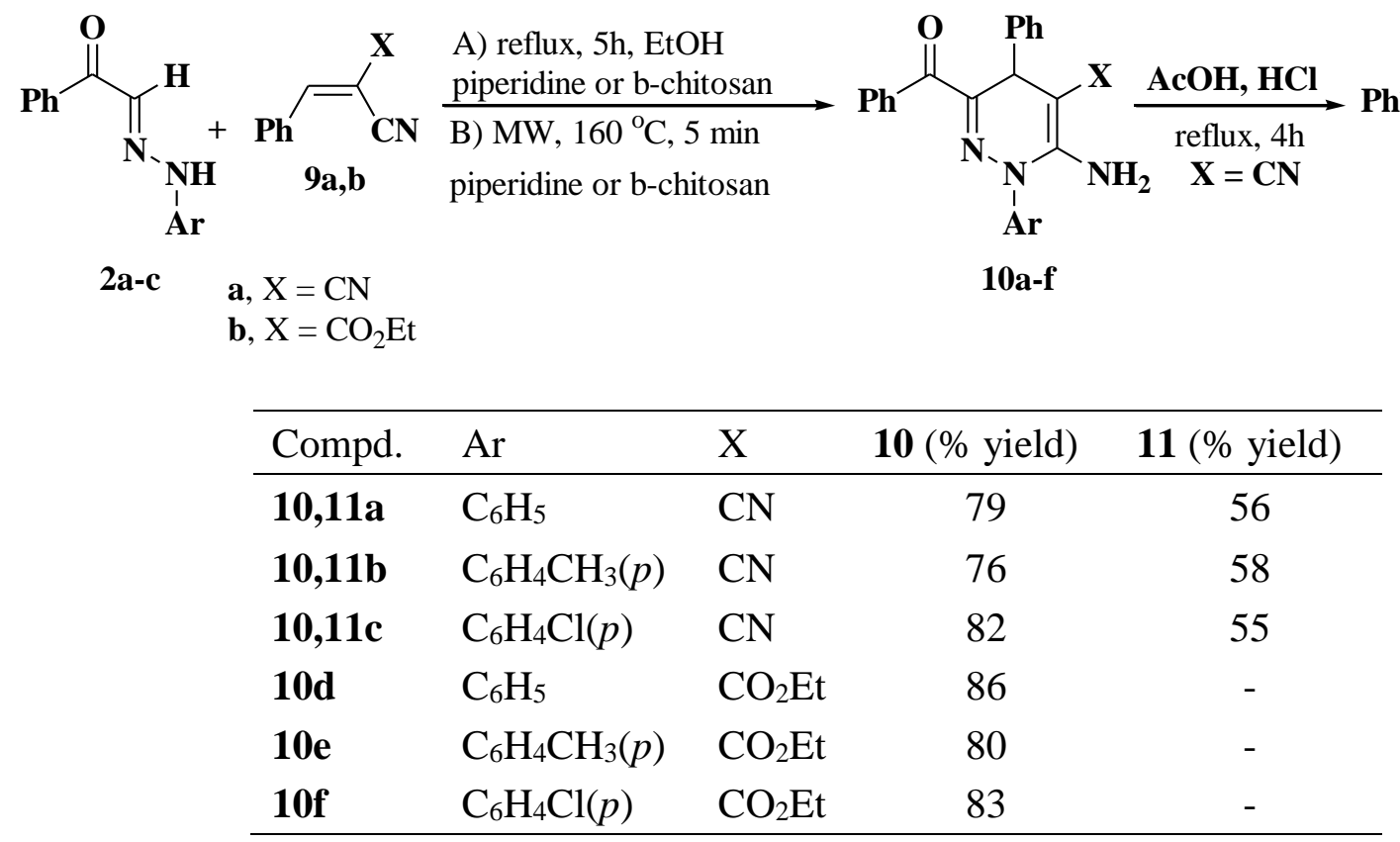

\section{Scheme 5}

\section{Conclusions}

The synthetic approach to arylhydrazonals desribed here is more efficient and more atom economic than the original approach of coupling enaminones with arylhydrzonium salts. The recently reported synthesis of pyridazines via Baylis-Hillman reaction of arylhydrazonals with electron poor alkenes has been extended and the cyclic intermediate adduct suggested for this reaction was isolated and fully characterized; this led to the synthesis of two new structural types, $\mathbf{8}$ and 11a-c. 


\section{Experimental Section}

General. Melting points were recorded on Gallenkamp apparatus. IR spectra were recorded in $\mathrm{KBr}$ disks on a Perkin Elmer System 2000 FT-IR spectrophotometer. ${ }^{1} \mathrm{H}$ and ${ }^{13} \mathrm{C}$ NMR spectra were recorded on a Bruker DPX $400 \mathrm{MHz}$ super-conducting NMR spectrometer; proton spectra were measured at $400 \mathrm{MHz}$ and carbon spectra at $100 \mathrm{MHz}$. IR data are in $\mathrm{cm}^{-1}$. Mass spectra were measured on VG Auto-spec-Q (high resolution, high performance, tri-sector GC/MS/MS) and with LCMS using Agilent 1100 series LC/MSD with an API-ES/APCI ionization mode. Microanalyses were performed on LECO CH NS-932 Elemental Analyzer. The microwave oven used was a single mode cavity Explorer Microwave (CEM Corporation, NC, USA) and irradiation was conducted in heavy-walled Pyrex tubes (capacity $10 \mathrm{~mL}$ ) fitted with a PCS cap.

\section{2-Arylhydrazono-1-phenylethanones (2a-c). General procedures}

The procedure described ${ }^{17}$ for the synthesis of pyruvaldehyde-1-phenylhydrazone was adopted. Thus, to a solution of potassium hydroxide $(3.5 \mathrm{~g}, 62 \mathrm{mmol})$ in water $(100 \mathrm{~mL})$ was added ethyl benzoylacetate $(8.7 \mathrm{~g}, 10 \mathrm{mmol})$. The mixture was stirred at room temperature for $24 \mathrm{~h}$, cooled to $0-5{ }^{\circ} \mathrm{C}$ and aqueous $\mathrm{HCl}(20 \mathrm{~mL}, 2.5 \mathrm{M})$ was added slowly. The solution was made basic by adding sodium acetate $(8.2 \mathrm{~g}, 100 \mathrm{mmol})$ in cooled water $(30 \mathrm{~mL})$, then gradually treated with stirring with the appropriate aromatic diazonium chlorides $(10 \mathrm{mmol})$. After stirring for $2 \mathrm{~h}$ at 0 $5{ }^{\circ} \mathrm{C}$ the yellow precipitate was collected and recrystallized from ethanol to give 2a-c.

1-Phenyl-2-phenylhydrazonoethanone (2a). Yield $10.5 \mathrm{~g}$ (94\%), yellow crystals from $\mathrm{EtOH} / \mathrm{H}_{2} \mathrm{O} \mathrm{mp} 146-148{ }^{\circ} \mathrm{C}$ (lit. ${ }^{18} \mathrm{mp} 148-150{ }^{\circ} \mathrm{C}$ ). ${ }^{1} \mathrm{H}$ NMR $\left(\mathrm{CDCl}_{3}\right)$ : 14.52 (br, $\left.1 \mathrm{H}, \mathrm{NH}\right), 8.02$ $(\mathrm{d}, 2 \mathrm{H}, J 7.6 \mathrm{~Hz}), 7.76(\mathrm{~s}, 1 \mathrm{H}), 7.63(\mathrm{t}, 1 \mathrm{H}, J 7.7 \mathrm{~Hz}), 7.51(\mathrm{t}, 2 \mathrm{H}, J 7.8 \mathrm{~Hz}), 7.41-7.33(\mathrm{~m}, 4 \mathrm{H})$, 7.09 (t, $1 \mathrm{H}, J$ 7.6 Hz). ${ }^{13} \mathrm{C} \mathrm{NMR}\left(\mathrm{CDCl}_{3}\right): 186.2,140.7,137.3,132.9,129.5,128.9,128.0,123.9$, 122.3, 114.9. Anal. calcd. for $\mathrm{C}_{14} \mathrm{H}_{12} \mathrm{~N}_{2} \mathrm{O}$ (224.3): C 74.98; H 5.39; N 12.49. Found: C 74.89; H $5.31 ; \mathrm{N} 12.40$.

1-Phenyl-2-p-tolylhydrazonoethanone (2b). Yield $11.0 \mathrm{~g}$ (92\%), yellow crystals from EtOH, mp 114-115 ${ }^{\circ} \mathrm{C}$ (lit. ${ }^{19} \mathrm{mp} 115-117^{\circ} \mathrm{C}$ ). ${ }^{1} \mathrm{H}$ NMR $\left(\mathrm{CDCl}_{3}\right.$ ): 14.58 (br, $\left.1 \mathrm{H}, \mathrm{NH}\right), 8.00$ (dd, $2 \mathrm{H}, J$ 8.4, 1.6 Hz), $7.72(\mathrm{~s}, 1 \mathrm{H}), 7.59$ (t, $1 \mathrm{H}, J 7.4 \mathrm{~Hz}), 7.52$ (t, 2H, $J .5 \mathrm{~Hz}), 7.27$ (d, 2H, $J 8.4 \mathrm{~Hz})$, $7.18(\mathrm{~d}, 2 \mathrm{H}, J 8.4 \mathrm{~Hz}), 2.33(\mathrm{~s}, 3 \mathrm{H}) .{ }^{13} \mathrm{C} \mathrm{NMR}\left(\mathrm{CDCl}_{3}\right): 186.2,140.3,137.4,133.7,132.7,130.0$, 128.8, 128.0, 121.8, 114.9, 20.9. Anal. calcd. for $\mathrm{C}_{15} \mathrm{H}_{14} \mathrm{~N}_{2} \mathrm{O}$ (238.3): C 75.61; H 5.92; N 11.76. Found: C 75.59; H 5.91; N 11.73.

2-p-Chlorophenylhydrazono-1-phenylethanone (2c). Yield $11.0 \mathrm{~g}(88 \%)$, yellow crystals from EtOH, mp 148-150 ${ }^{\circ} \mathrm{C}$ (lit. ${ }^{20} \mathrm{mp} 147-148{ }^{\circ} \mathrm{C}$ ). IR: 3224, 3055, 1631, 1595, 1502, 1475, 1240, 1089, 1045, 821. ${ }^{1} \mathrm{H}$ NMR (DMSO-d $\left.\mathrm{d}_{6}\right): 11.59$ (br, 1H, NH), 7.97 (d, 2H, J 7.4 Hz), 7.72 (s, 1H), $7.62(\mathrm{t}, 1 \mathrm{H}, J 7.6 \mathrm{~Hz}), 7.54(\mathrm{t}, 2 \mathrm{H}, J 7.8 \mathrm{~Hz}), 7.36(\mathrm{~d}, 2 \mathrm{H}, J 8.8 \mathrm{~Hz}), 7.01(\mathrm{~d}, 2 \mathrm{H}, J 8.8 \mathrm{~Hz}) .{ }^{13} \mathrm{C}$ NMR ( $\left.\mathrm{CDCl}_{3}\right): 189.9,142.9,137.9,135.0,132.8,130.1,130.0,128.9,125.8,115.8$. MS: $\mathrm{m} / \mathrm{z}$ (\%) $260(\mathrm{M}+2,20), 258\left(\mathrm{M}^{+}, 25\right), 105$ (100). Anal. calcd. for $\mathrm{C}_{14} \mathrm{H}_{11} \mathrm{ClN}_{2} \mathrm{O}$ (258.7): $\mathrm{C} 65.00 ; \mathrm{H}$ 4.29; N 10.83. Found: C 64.79; H 4.21; N 10.73. 


\section{2-Arylhydrazono-3-hydroxy-1-phenylpropan-1-ones (3a-c). General procedures}

Method A. A mixture of each of 2 a-c $(10.0 \mathrm{mmol})$ and excess aqueous formaldehyde $(3.2 \mathrm{~g}$, $40.0 \mathrm{mmol}, 37 \%)$ in methanol $(20 \mathrm{~mL})$, TEA (5 drops) was stirred at r.t. over night. After evaporation the solvent in vacuum, ice water $(50 \mathrm{~mL})$ was added and the yellow precipitate formed was collected by filtration and crystallized from the proper solvent to give 3a-c in 70$75 \%$ yield

Method B. A mixture of each of $2 \mathbf{a}-\mathbf{c}(10.0 \mathrm{mmol})$ and aqueous formaldehyde $(0.8 \mathrm{~g}, 10.0$ mmol, 37\%) was refluxed for 2 hours in methanol in presence of montmorilonite K10. After reflux, evaporation the solvent, ice water $(50 \mathrm{~mL})$ was added and the yellow precipitate formed was collected by filtration and crystallized to give 3a-c in $90-95 \%$ yield.

3-Hydroxy-1-phenyl-2-phenylhydrazonopropan-1-one (3a). Yield 1.8 g (70\%), method A, $2.30 \mathrm{~g}(90 \%)$, method $\mathrm{B}$, yellow crystals from benzene, mp 128-130 ${ }^{\circ} \mathrm{C}$ (lit. ${ }^{21} \mathrm{mp} 126-127{ }^{\circ} \mathrm{C}$ ). IR: 3448, 3305, 3056, 2965, 1630, 1547, 1492, 1367, 1257, 986. ${ }^{1} \mathrm{H}$ NMR (DMSO-d 6$): 10.64$ (s, 1H, NH), $7.81(\mathrm{~d}, 2 \mathrm{H}, J 7.2 \mathrm{~Hz}), 7.58(\mathrm{t}, 1 \mathrm{H}, J 7.4 \mathrm{~Hz}), 7.50(\mathrm{t}, 2 \mathrm{H}, J 7.6 \mathrm{~Hz}), 7.27$ (t, 2H, J 7.8 $\mathrm{Hz}), 7.16(\mathrm{~d}, 2 \mathrm{H}, J 7.6 \mathrm{~Hz}), 6.93(\mathrm{t}, 1 \mathrm{H}, J 7.2 \mathrm{~Hz}), 5.20(\mathrm{br}, 1 \mathrm{H}, \mathrm{OH}), 4.64\left(\mathrm{~s}, 2 \mathrm{H}, \mathrm{CH}_{2}\right) .{ }^{13} \mathrm{C}$ NMR (DMSO-d ${ }_{6}$ ): 190.9, 143.6, 141.1, 138.4, 131.2, 129.9, 129.3, 127.7, 121.8, 114.1, 53.5. LCMS $m / z=255(\mathrm{M}+1)$. MS: $m / z(\%) 254\left(\mathrm{M}^{+}, 10\right), 229$ (70), 182 (25), 91 (100). Anal. calcd. for $\mathrm{C}_{15} \mathrm{H}_{14} \mathrm{~N}_{2} \mathrm{O}_{2}$ (254.3): C 70.85; H 5.55; N 11.02. Found: C 70.74; H 5.51; N 11.03.

3-Hydroxy-1-phenyl-2-p-tolylhydrazonopropan-1-one (3b). Yield $2.0 \mathrm{~g}$ (75\%), method A, $2.45 \mathrm{~g}(91 \%)$, method B, yellow crystals from $\mathrm{EtOH} / \mathrm{H}_{2} \mathrm{O}, \mathrm{mp} 164-165{ }^{\circ} \mathrm{C}$. IR: $3438,3184,3056$, 2961, 1638, 1566, 1515, 1449, 1240, 968, 728. ${ }^{1} \mathrm{H}$ NMR (DMSO-d ): $_{10.55}$ (br, 1H, NH), 7.79 $(\mathrm{dd}, 2 \mathrm{H}, J$ 8.4, $1.4 \mathrm{~Hz}), 7.52$ (t, 1H, J 8.0 Hz), 7.49 (t, 2H, J 7.2 Hz), $7.08(\mathrm{~m}, 4 \mathrm{H}), 5.30(\mathrm{br}, 1 \mathrm{H}$, $\mathrm{OH}), 4.63$ (s, 2H, $\mathrm{CH}_{2}$ ), 2.51 (s, 3H, $\left.\mathrm{CH}_{3}\right) .{ }^{13} \mathrm{C}$ NMR (DMSO-d $)$ ): 190.7, 141.3, 140.5, 138.5, 131.1, 130.7, 129.9, 129.7, 127.6, 114.1, 52.6, 20.3. LCMS $m / z=269(\mathrm{M}+1)$. Anal. calcd. for $\mathrm{C}_{16} \mathrm{H}_{16} \mathrm{~N}_{2} \mathrm{O}_{2}$ (268.3): C 71.62; H 6.01; N 10.44. Found: C 71.54; H 6.00; N 10.42.

2-p-Chlorophenyhydrazono-3-hydroxy-1-phenylpropan-1-one (3c). Yield 2.1g (73\%), method A, $2.7 \mathrm{~g}(94 \%)$, method B, yellow crystals from benzene, mp 166-168 ${ }^{\circ} \mathrm{C}$ (lit. ${ }^{22} \mathrm{mp} 166-$ $167{ }^{\circ} \mathrm{C}$ ). IR: 3379, 3307, 3056, 2969, 1633, 1551, 1486, 1362, 1251, 968, 728. ${ }^{1} \mathrm{H}$ NMR (DMSOd $)) 10.75(\mathrm{br}, 1 \mathrm{H}, \mathrm{NH}), 7.80(\mathrm{~d}, 2 \mathrm{H}, J 7.8 \mathrm{~Hz}), 7.56(\mathrm{t}, 1 \mathrm{H}, J 7.6 \mathrm{~Hz}), 7.50(\mathrm{t}, 2 \mathrm{H}, J 7.4 \mathrm{~Hz}), 7.32$ $(\mathrm{d}, 2 \mathrm{H}, J 8.8 \mathrm{~Hz}), 7.16(\mathrm{~d}, 2 \mathrm{H}, J 8.8 \mathrm{~Hz}), 5.33(\mathrm{br}, 1 \mathrm{H}, \mathrm{OH}), 4.61\left(\mathrm{~s}, 2 \mathrm{H}, \mathrm{CH}_{2}\right) .{ }^{13} \mathrm{C} \mathrm{NMR}$ $\left(\mathrm{CDCl}_{3}\right): 191.2,141.6,139.6,137.4,131.8,130.5,129.4,127.8,127.5,115.5,57 . \mathrm{MS}: \mathrm{m} / \mathrm{z}(\%)$ $288\left(\mathrm{M}^{+}, 20\right), 202$ (50), 139 (85). Anal. calcd for $\mathrm{C}_{15} \mathrm{H}_{13} \mathrm{ClN}_{2} \mathrm{O}_{2}$ (288.7): $\mathrm{C}$ 62.40; H 4.54; N 9.70. Found: C 62.24; H 4.50; N 9.59.

\section{2-Arylhydrazono-3-oxo-3-phenylpropanals (1a-c). General procedure}

A mixture of each of 3a-c $(10.0 \mathrm{mmol})$ in nitrobenzene $(10 \mathrm{~mL})$ was refluxed for $2 \mathrm{~h}$ or irradiated in a microwave oven for $2 \mathrm{~min}$ at $140{ }^{\circ} \mathrm{C}$. After cooling, petroleum ether $(50 \mathrm{~mL})$ was added and the solid formed was collected by filtration and recrystallized from ethanol to yield 1a-c in $65-70 \%$ yield. 
3-Oxo-3-phenyl-2-phenylhydrazonopropanal (1a). Yield $1.64 \mathrm{~g}(65 \%)$, yellow crystals from EtOH, mp 82-83 ${ }^{\circ} \mathrm{C}$ (lit. $\left.{ }^{15} \mathrm{mp} .82-84{ }^{\circ} \mathrm{C}\right)$. LCMS: $m / z=253(\mathrm{M}+1)$.

3-Oxo-3-phenyl-2-p-tolylhydrazonopropanal (1b). Yield $1.8 \mathrm{~g}(67 \%)$, red crystals from EtOH, mp 116-118 ${ }^{\circ} \mathrm{C}$ (lit. $\left.{ }^{15} \mathrm{mp} 115-17^{\circ} \mathrm{C}\right)$. LCMS: $m / z=267(\mathrm{M}+1)$

2-p-Chlorophenylhydrazono-3-oxo-3-phenylpropanal (1c). Yield $2.0 \mathrm{~g}(70 \%)$, red crystals from EtOH, mp 142-144 ${ }^{\circ} \mathrm{C}$ (lit. $\left.{ }^{15} \mathrm{mp} 143-45^{\circ} \mathrm{C}\right)$. LCMS: $m / z=286(\mathrm{M}+1), 288(\mathrm{M}+2)$

1-(6-Benzoyl-5-hydroxy-2-phenyl-2,3,4,5-tetrahydropyridazin-4-yl)ethanone (5). A mixture of compound $1 \mathrm{a}(0.50 \mathrm{~g}, 2.0 \mathrm{mmol})$, methyl vinyl ketone $(0.3 \mathrm{~g}, 4.0 \mathrm{mmol}$, ) and DABCO $(0.224$ $\mathrm{g}, 2.0 \mathrm{mmol}$ ) was mixed then placed in a microwave oven and irradiated at $100{ }^{\circ} \mathrm{C}$ for $1 \mathrm{~min}$. The mixture was then washed with hot petroleum ether (60-80) and crystallized from ethanol to give 5. Yield $0.50 \mathrm{~g}(78 \%)$ yellow crystals from $\mathrm{EtOH}, \mathrm{mp} 215-217^{\circ} \mathrm{C}$. IR: 3359, 3063, 2906, 1704, $1627,1549,1493,1334,1271,1222,1175,1065,957,876 .{ }^{1} \mathrm{H}$ NMR $\left(\mathrm{CDCl}_{3}\right): 8.00$ (dd, $2 \mathrm{H}, J$ 7.8, $1.6 \mathrm{~Hz}), 7.56(\mathrm{t}, 1 \mathrm{H}, J 7.6 \mathrm{~Hz}), 7.48(\mathrm{t}, 2 \mathrm{H}, J 7.4 \mathrm{~Hz}), 7.37(\mathrm{~m}, 4 \mathrm{H}), 7.11(\mathrm{~m}, 1 \mathrm{H}), 5.36(\mathrm{~m}$, 1H), 4.23 (dd, 1H, J 13.2 Hz), 3.74 (t, 1H, J 13.2 Hz), 3.13 (br, 1H, OH), 2.88 (dd, 1H, J 13.2 $\mathrm{Hz}), 2.45$ (s, 3H). ${ }^{13} \mathrm{C} \mathrm{NMR}\left(\mathrm{CDCl}_{3}\right): 204.7,191.5,145.6,139.5,137.2,131.9,130.5,129.3$, 127.9, 123.8, 116.1, 57.6, 46.8, 39.6, 28.5. MS: m/z (\%) $322\left(\mathrm{M}^{+}, 60\right), 304$ (20), 105 (85). Anal. calcd. for $\mathrm{C}_{19} \mathrm{H}_{18} \mathrm{~N}_{2} \mathrm{O}_{3}$ (322.4): C 70.79; H 5.63; N 8.69. Found: C 70.52; H 5.63; N 8.66.

1-(6-Benzoyl-2-phenyl-2,3-dihydropyridazine-4-yl)ethanone (6). A mixture of 5 (0.32 g, 1.0 $\mathrm{mmol})$, in acetic acid $(5 \mathrm{~mL})$, was placed in a microwave and irradiated at $160{ }^{\circ} \mathrm{C}$ for $5 \mathrm{~min}$. After cooling ice water $(10 \mathrm{~mL})$ was added and the yellow precipitate so formed was collected and crystallized from ethanol to give 6 in $0.28 \mathrm{~g}(93 \%)$, mp.160-162 ${ }^{\circ} \mathrm{C}$ (lit. ${ }^{15} \mathrm{mp} 160-162{ }^{\circ} \mathrm{C}$ ). ${ }^{1} \mathrm{H}$ NMR $\left(\mathrm{CDCl}_{3}\right): 8.00(\mathrm{~d}, 2 \mathrm{H}, J 8.0 \mathrm{~Hz}), 7.69(\mathrm{~s}, 1 \mathrm{H}), 7.57$ (t, 1H, J 7.8 Hz), 7.48 (t, 2H, J 7.6 Hz), $7.42(\mathrm{~m}, 4 \mathrm{H}), 7.22(\mathrm{~m}, 1 \mathrm{H}), 4.74(\mathrm{~s}, 2 \mathrm{H}), 2.54(\mathrm{~s}, 3 \mathrm{H}) .{ }^{13} \mathrm{C} \mathrm{NMR}\left(\mathrm{CDCl}_{3}\right)$ 196.7, 189.0, 144.5, 137.1, 136.5, 132.0, 130.5, 129.3, 127.9, 127.5, 124.9, 123.3, 116.4, 43.7, 25.2. Anal. calcd. for $\mathrm{C}_{19} \mathrm{H}_{16} \mathrm{~N}_{2} \mathrm{O}_{2}$ (304.4): C 74.93; H 5.30; N 9.20. Found: C 74.85; H 5.23; N 9.16. Crystal data: $\mathrm{a}=$ 9.7094(3), $b=10.0166(3), c=16.0286(12) \AA ; \alpha=82.833(6), \beta=86.536(6), \gamma=84.749(6)^{\circ}$; space group P1; CCDC number 805703.

3-Benzoyl-1,6-diphenyl-1,2-dihydropyrrolo[3,4-c]pyridazine-5,7-dione (8). A mixture of compound 1a $(0.225 \mathrm{~g}, 1.0 \mathrm{mmol}), N$-phenylmaleimide $(0.173 \mathrm{~g}, 1.0 \mathrm{mmol}), \mathrm{DABCO}(0.224 \mathrm{~g}$, $2.0 \mathrm{mmol})$ and diphenyl ether $(2.0 \mathrm{~g})$ were microwave irradiated at $200{ }^{\circ} \mathrm{C}$ for $5 \mathrm{~min}$. The mixture was then washed with hot petroleum ether (60-80) and crystallized from DMF to give 8 as yellow crystals, yield $0.30 \mathrm{~g}(73 \%)$, mp 253-255 ${ }^{\circ} \mathrm{C}$. IR: $3359,3063,2906,1704,1627,1549$, 1493, 1334, 1271, 1222, 1175, 1065, 957, 876. ${ }^{1} \mathrm{H}$ NMR $\left(\mathrm{CDCl}_{3}\right) 10.45$ (br, 1H, NH), 9.70 (s, 1H), $7.90(\mathrm{~d}, 2 \mathrm{H}, J 7.6 \mathrm{~Hz}), 7.80(\mathrm{~d}, 2 \mathrm{H}, J 7.8 \mathrm{~Hz}), 7.61(\mathrm{tt}, 2 \mathrm{H}, J$ 7.8, $1.2 \mathrm{~Hz}), 7.56-7.48(\mathrm{~m}$, $6 \mathrm{H}), 7.39(\mathrm{~m}, 2 \mathrm{H}), 7.31(\mathrm{tt}, 1 \mathrm{H}, J 7.8,1.2 \mathrm{~Hz}) .{ }^{13} \mathrm{C} \mathrm{NMR}\left(\mathrm{CDCl}_{3}\right): 195.6,171.3,168.4,137.2$, 131.9, 129.7, 129.1, 128.3, 127.7, 126.8, 126.3, 125.7, 125.5, 123.8, 121.9, 121.5, 119.4, 101.7, 96.9. LCMS: $m / z=408(\mathrm{M}+1)$. MS: $m / z(\%) 407\left(\mathrm{M}^{+}, 100\right), 379$ (20), 287 (25). Anal. calcd. for $\mathrm{C}_{25} \mathrm{H}_{17} \mathrm{~N}_{3} \mathrm{O}_{3}$ (407.4): $\mathrm{C} 73.70 ; \mathrm{H} 4.21 ; \mathrm{N} \mathrm{10.31.} \mathrm{Found:} \mathrm{C} 73.59 ; \mathrm{H}$ 4.13; $\mathrm{N}$ 10.26. HRMS = $407.1264\left(\mathrm{C}_{25} \mathrm{H}_{17} \mathrm{~N}_{3} \mathrm{O}_{3}\right.$ requires 407.1264$)$ 


\section{3-Amino-6-benzoyl-2,5-dihydropyridazine derivatives (10a-f). General procedures}

Method A. A mixture of each of $\mathbf{2 a - c}(2.0 \mathrm{mmol})$ and benzylidenemalononitrile and/or ethyl 2cyanocinnamate $(2.0 \mathrm{mmol})$ in ethanol $(20 \mathrm{~mL})$ and piperidine $(5$ drops $)$ or $\beta$-chitosan $(0.2 \mathrm{~g}, 13$ mmol) was refluxed for 5 hours, the mixture was then poured into ice water $(50 \mathrm{~mL})$ and acidified with conc. $\mathrm{HCl}(2 \mathrm{~mL})$. The yellow precipitate so formed was collected by filtration and crystallized from ethanol to yield compounds 10a-f.

Method B. A mixture of each of $\mathbf{2 a - c}(2.0 \mathrm{mmol})$, and benzylidenemalononitrile and/or ethyl 2cyanocinnamate $(2.0 \mathrm{mmol})$ in dioxane $(5 \mathrm{~mL})$ and piperidine $(5 \mathrm{drops})$ or $\beta$-chitosan $(0.2 \mathrm{~g}, 13$ mmol) was mixed and placed in a microwave oven and irradiated at $160{ }^{\circ} \mathrm{C}$ for 5 minutes. The mixture was then poured into ice water $(50 \mathrm{~mL})$ and acidified with conc. $\mathrm{HCl}(2 \mathrm{~mL})$. The yellow precipitate was collected and crystallized from ethanol.

3-Amino-6-benzoyl-2,5-diphenyl-2,5-dihydropyridazine-4-carbonitrile (10a). Yield $0.56 \mathrm{~g}$ (74\%) method A, $0.6 \mathrm{~g}$ (79\%) method B, yellow crystals from EtOH, mp 163-164 ${ }^{\circ} \mathrm{C}$. IR: 3456, 3336, 3059, 2185, 1637, 1593, 1568, 1491, 1410, 1247, 1141. ${ }^{1} \mathrm{H}$ NMR $\left(\mathrm{CDCl}_{3}\right): 7.92$ (dd, 2H, $J$ 7.6, $1.2 \mathrm{~Hz}), 7.53-7.34(\mathrm{~m}, 13 \mathrm{H}), 5.27(\mathrm{~s}, 1 \mathrm{H}), 4.37$ (br, $\left.2 \mathrm{H}, \mathrm{NH}_{2}\right) .{ }^{13} \mathrm{C} \mathrm{NMR}\left(\mathrm{CDCl}_{3}\right): 190.3$, 149.4, 145.1, 141.0, 139.6, 136.2, 132.7, 130.5, 129.8, 129.2, 128.4, 128.0, 127.7, 127.4, 125.4, 119.8, 60.0, 37.0. LCMS: $m / z=379(\mathrm{M}+1)$. MS: $m / z(\%) 378\left(\mathrm{M}^{+}, 10\right), 301$ (25), 273 (100). Anal. calcd. for $\mathrm{C}_{24} \mathrm{H}_{18} \mathrm{~N}_{4} \mathrm{O}$ (378.4): C 76.17; H 4.79; N 14.80. Found: C 76.12; H 4.79; N 14.65. 3-Amino-6-benzoyl-5-phenyl-2-p-tolyl-2,5-dihydropyridazine-4-carbonitrile (10b). Yield $0.57 \mathrm{~g}(73 \%)$ method A, $0.6 \mathrm{~g}(76 \%)$ method B, yellow crystals from EtOH, mp 198-200 ${ }^{\circ} \mathrm{C}$. IR: 3454, 3323, 3056, 2181, 1649, 1599, 1565, 1509, 1403, 1250, 1128, 872, 718, 698. ${ }^{1} \mathrm{H}$ NMR $\left(\mathrm{CDCl}_{3}\right): 7.92(\mathrm{dd}, 2 \mathrm{H}, J$ 8.4, $1.2 \mathrm{~Hz}), 7.49(\mathrm{tt}, 1 \mathrm{H}, J$ 7.6, $1.2 \mathrm{~Hz}), 7.39-7.27(\mathrm{~m}, 11 \mathrm{H}), 5.25(\mathrm{~s}$, 1H), 4.39 (br, 2H, $\left.\mathrm{NH}_{2}\right), 2.42\left(\mathrm{~s}, 3 \mathrm{H}, \mathrm{CH}_{3}\right) .{ }^{13} \mathrm{C} \mathrm{NMR}\left(\mathrm{CDCl}_{3}\right)$ : 190.3, 149.6, 144.8, 141.2, 138.7, 137.1, 136.3, 132.5, 130.5, 130.4, 129.1, 127.9, 127.6, 127.2, 125.5, 119.9, 59.6, 37.0, 21.1. LCMS: $m / z=393(\mathrm{M}+1)$. MS: $m / z(\%) 392\left(\mathrm{M}^{+}, 20\right), 315$ (45), 287 (100), 105 (75). Anal. calcd. for $\mathrm{C}_{25} \mathrm{H}_{20} \mathrm{~N}_{4} \mathrm{O}$ (392.5): C 76.51; H 5.14; N 14.28. Found: C 76.42; H 5.19; N 14.25.

3-Amino-6-benzoyl-2-p-chlorophenyl-5-phenyl-2,5-dihydropyridazine-4-carbonitrile (10c). Yield $0.60 \mathrm{~g}$ (73\%) method A, $0.68 \mathrm{~g}$ (82\%) method B, yellow crystals from EtOH, mp 203-204 ${ }^{\circ} \mathrm{C}$. IR: 3469, 3324, 3059, 2189, 1641, 1592, 1567, 1488, 1423, 1245, 1142, 873, 700. ${ }^{1} \mathrm{H}$ NMR $\left(\mathrm{CDCl}_{3}\right): 7.90(\mathrm{dd}, 2 \mathrm{H}, J$ 8.4, $1.2 \mathrm{~Hz}), 7.53(\mathrm{tt}, 1 \mathrm{H}, J$ 7.6, $1.2 \mathrm{~Hz}), 7.48-7.29(\mathrm{~m}, 11 \mathrm{H}), 5.26(\mathrm{~s}$, 1H), 4.42 (br, $\left.2 \mathrm{H}, \mathrm{NH}_{2}\right) .{ }^{13} \mathrm{C} \mathrm{NMR}\left(\mathrm{CDCl}_{3}\right)$ : 190.2, 149.3, 145.5, 140.7, 138.1, 136.1, 134.1, 132.7, 130.4, 129.9, 129.2, 128.0, 127.8, 127.1, 126.6, 119.6, 60.4, 37.2. LCMS: $m / z=413(\mathrm{M}+$ 1). MS: $m / z(\%) 414(\mathrm{M}+2,10), 412\left(\mathrm{M}^{+}, 10\right), 335$ (25), 307 (100), 105. Anal. calcd. for $\mathrm{C}_{24} \mathrm{H}_{17} \mathrm{ClN}_{4} \mathrm{O}$ (412.9): C 69.82; H 4.15; N 13.57. Found: C 69.72; H 4.19; N 13.45.

3-Amino-6-benzoyl-2,5-diphenyl-2,5-dihydropyridazine-4-carboxylic acid ethyl ester (10d). Yield $0.66 \mathrm{~g}$ (77\%), method A, $0.73 \mathrm{~g}(86 \%)$, method B, yellow crystals from EtOH, mp 168$170{ }^{\circ} \mathrm{C}$. IR: $3477,3275,3053,1665,1627,1515,1479,1373,1271,1125,1094 .{ }^{1} \mathrm{H}$ NMR $\left(\mathrm{CDCl}_{3}\right): 7.93(\mathrm{~d}, 2 \mathrm{H}, J 7.8 \mathrm{~Hz}), 7.49(\mathrm{~m}, 5 \mathrm{H}), 7.39(\mathrm{~m}, 5 \mathrm{H}), 7.29(\mathrm{~m}, 2 \mathrm{H}), 7.21(\mathrm{t}, 1 \mathrm{H}, J 7.8 \mathrm{~Hz})$, 6.28 (br, 2H, $\left.\mathrm{NH}_{2}\right), 5.61(\mathrm{~s}, 1 \mathrm{H}), 4.19(\mathrm{q}, 2 \mathrm{H}, J 7.0 \mathrm{~Hz}), 1.31(\mathrm{t}, 3 \mathrm{H}, J 7.0 \mathrm{~Hz}) .{ }^{13} \mathrm{C} \mathrm{NMR}$ $\left(\mathrm{CDCl}_{3}\right): 190.9,169.2,150.3,147.9,142.9,139.8,136.7,132.3,130.5,129.6,128.6,127.9$, 
127.8, 127.6, 126.8, 125.5, 77.9, 59.6, 35.9, 14.5. LCMS: $m / z=426(\mathrm{M}+1) . \mathrm{MS}: m / z(\%) 425$ $\left(\mathrm{M}^{+}, 15\right), 352$ (100), 320 (70). Anal. calcd. for $\mathrm{C}_{26} \mathrm{H}_{23} \mathrm{~N}_{3} \mathrm{O}_{3}$ (425.5): C 73.40; H 5.45; N 9.88. Found: C 73.42; H 5.39; N 9.85.

3-Amino-6-benzoyl-5-phenyl-2-p-tolyl-2,5-dihydropyridazine-4-carboxylic acid ethyl ester (10e). Yield $0.65 \mathrm{~g}(74 \%)$, method A, $0.70 \mathrm{~g}$ (80\%), method B, yellow crystals from EtOH, mp 188-190 ${ }^{\circ} \mathrm{C}$. IR: 3482, 3302, 3058, 1656, 1626, 1507, 1453, 1371, 1243, 1126, 1094, 898. ${ }^{1} \mathrm{H}$ NMR $\left(\mathrm{CDCl}_{3}\right): 7.92(\mathrm{dd}, 2 \mathrm{H}, J$ 8.4, $1.2 \mathrm{~Hz}), 7.49$ (tt, $1 \mathrm{H}, J$ 7.6, 1.2 Hz), 7.39-7.34 (m, 5H), 7.317.27 (m, 5H), 7.21 (tt, 1H, J 7.8, 1.2 Hz), 6.27 (br, 2H, NH 2$), 5.60$ (s, 1H), 4.18 (q, 2H, J 7.0 Hz), 2.44, (s, 3H), 1.31 (t, 3H, J 7.0 Hz). ${ }^{13} \mathrm{C} \mathrm{NMR}\left(\mathrm{CDCl}_{3}\right): 191.0,169.3,150.4,147.6,143.1,138.1$, 137.3, 136.8, 132.2, 130.5, 130.3, 128.6, 127.8, 127.6, 126.8, 125.6, 77.6, 59.6, 35.9, 21.1, 14.5. LCMS: $m / z=440(\mathrm{M}+1)$. MS: $m / z(\%) 439\left(\mathrm{M}^{+}, 20\right), 366(100), 334$ (70), 105 (85). Anal. calcd. for $\mathrm{C}_{27} \mathrm{H}_{25} \mathrm{~N}_{3} \mathrm{O}_{3}$ (439.5): C 73.79; H 5.73; N 9.56. Found: C 73.62; H 5.69; N 9.55.

3-Amino-6-benzoyl-2-p-chlorophenyl-5-phenyl-2,5-dihydropyridazine-4-carboxylic acid ethyl ester (10f). Yield $0.70 \mathrm{~g}(76 \%)$ method A, $0.76 \mathrm{~g}(83 \%)$ method B, yellow crystals from EtOH, mp 176-178 ${ }^{\circ} \mathrm{C}$. IR: 3483, 3285, 3059, 1658, 1618, 1493, 1370, 1242, 1125, 1083, 895, 704. ${ }^{1} \mathrm{H} \mathrm{NMR}\left(\mathrm{CDCl}_{3}\right): 7.89$ (dd, $2 \mathrm{H}, J$ 8.4, $\left.1.2 \mathrm{~Hz}\right), 7.51(\mathrm{t}, 1 \mathrm{H}, J 7.4 \mathrm{~Hz}), 7.44(\mathrm{~m}, 3 \mathrm{H}), 7.43-$ $7.34(\mathrm{~m}, 4 \mathrm{H}), 7.29(\mathrm{~m}, 3 \mathrm{H}), 7.21(\mathrm{t}, 1 \mathrm{H}, J 7.2 \mathrm{~Hz}), 6.24\left(\mathrm{br}, 2 \mathrm{H}, \mathrm{NH}_{2}\right), 5.62(\mathrm{~s}, 1 \mathrm{H}), 4.18(\mathrm{q}, 2 \mathrm{H}$, $J 7.0 \mathrm{~Hz}), 1.31(\mathrm{t}, 3 \mathrm{H}, J 7.0 \mathrm{~Hz}) .{ }^{13} \mathrm{C} \mathrm{NMR}\left(\mathrm{CDCl}_{3}\right): 190.9,169.1,150.0,148.3,142.6,138.3$, 136.6, 133.5, 132.4, 130.4, 129.8, 128.7, 127.9, 127.5, 126.9, 126.7, 78.4, 59.8, 36.0, 14.5. MS: $m / z(\%) 461\left(\mathrm{M}^{+}+2,10\right), 460\left(\left(\mathrm{M}^{+}+1,30\right), 386\right.$ (30), 270 (100). Anal. calcd. for $\mathrm{C}_{26} \mathrm{H}_{22} \mathrm{ClN}_{3} \mathrm{O}_{3}$ (459.9): C 67.90; H 4.82; N 9.14. Found: C 67.88; H 4.79; N 9.15.

\section{6-Benzoyl-3-oxo-2,3-dihydopyridazine-4-carbonitrile (11a-c). General procedure}

A mixture of each of 10a-c $(2.0 \mathrm{mmol})$ in acetic acid $(10 \mathrm{~mL})$ and hydrochloric acid $(5 \mathrm{~mL})$ was refluxed for $4 \mathrm{~h}$. The mixture was then poured into ice water $(50 \mathrm{~mL})$ and the yellow precipitate so formed was collected by filtration and recrystallized from ethanol to give 11a-c.

6-Benzoyl-3-oxo-2,5-diphenyl-2,3-dihydopyridazine-4-carbonitrile (11a). Yield $0.42 \mathrm{~g}$ (56\%) yellow crystals from EtOH, mp 258-260 ${ }^{\circ} \mathrm{C}$. IR: 3057, 3028, 2231, 1671, 1595, 1492, 1447 , 1372, 1327, 1303, 1229, 1192, 1166, 1088, 990, 911, 869. ${ }^{1} \mathrm{H}$ NMR $\left(\mathrm{CDCl}_{3}\right): 7.93$ (dd, 2H, J 8.4, $1.4 \mathrm{~Hz}), 7.69(\mathrm{dd}, 2 \mathrm{H}, J$ 8.0, $1.2 \mathrm{~Hz}), 7.65(\mathrm{t}, 2 \mathrm{H}, J 7.8 \mathrm{~Hz}), 7.54-7.39(\mathrm{~m}, 9 \mathrm{H})) .{ }^{13} \mathrm{C}$ NMR $\left(\mathrm{CDCl}_{3}\right): 188.5,164.2,156.1,151.1,143.6,140.0,135.0,134.6,131.2,131.0,130.4,129.2$, 129.1, 128.9, 128.8, 128.1, 124.9, 114.9. MS: m/z (\%) 377 ( $\left.\mathrm{M}^{+}, 75\right), 348$ (10), 105 (100). Anal. calcd. for $\mathrm{C}_{24} \mathrm{H}_{15} \mathrm{~N}_{3} \mathrm{O}_{2}$ (377.4): C 76.38; $\mathrm{H} 4.01 ; \mathrm{N} 11.13$. Found: C 76.32; H 3.99; N 11.15.

6-Benzoyl-3-oxo-5-phenyl-2-p-tolyl-2,3-dihydropyridazine-4-carbonitrile (11b). Yield $0.46 \mathrm{~g}$ (59\%) yellow crystals from EtOH, mp 228-230 ${ }^{\circ} \mathrm{C}$. IR: 3065, 3035, 2199, 1667, 1597, 1566, 1493, 1449, 1327, 1191, 1166, 870. ${ }^{1} \mathrm{H}$ NMR $\left(\mathrm{CDCl}_{3}\right): 7.92$ (dd, 2H, J 8.0, $\left.1.4 \mathrm{~Hz}\right), 7.63$ (t, 1H, $J$ $7.8 \mathrm{~Hz}), 7.58-7.44(\mathrm{~m}, 3 \mathrm{H}), 7.43-7.37(\mathrm{~m}, 6 \mathrm{H}), 7.30(\mathrm{~d}, 2 \mathrm{H}, J 8.0 \mathrm{~Hz}), 2.42\left(\mathrm{~s}, 3 \mathrm{H}, \mathrm{CH}_{3}\right) .{ }^{13} \mathrm{C}$ NMR $\left(\mathrm{CDCl}_{3}\right)$ : 188.5, 164.5, 156.1, 150.9, 143.4, 137.6, 135.7, 135.1, 134.1, 131.1, 130.4, 130.3, 129.6, 129.1, 128.8, 128.1, 124.6, 114.7, 21.2. MS: $m / z(\%) 391\left(\mathrm{M}^{+}, 30\right), 366(25), 105$ 
(100). Anal. calcd. for $\mathrm{C}_{25} \mathrm{H}_{17} \mathrm{~N}_{3} \mathrm{O}_{2}$ (391.4): C 76.71; H 4.38; N 10.73. Found: C 76.62; H 4.29; N 10.65 .

6-Benzoyl-2-p-chlorophenyl-3-oxo-5-phenyl-2,3-dihydropyridazine-4-carbonitrile

(11c). Yield $0.45 \mathrm{~g}(55 \%)$, yellow crystals from EtOH, mp 236-238 ${ }^{\circ} \mathrm{C}$. IR: 3066, 3038, 2229, 1668, 1596, 1490, 1449, 1328, 1192, 1167, 1092, 834, 759, 688. ${ }^{1} \mathrm{H} \mathrm{NMR}\left(\mathrm{CDCl}_{3}\right): 7.99$ (dd, 2H, $J$ 8.4, $1.4 \mathrm{~Hz}), 7.67(\mathrm{~m}, 2 \mathrm{H}), 7.56-7.39(\mathrm{~m}, 9 \mathrm{H}), 7.31\left(\mathrm{t}, 1 \mathrm{H}, J\right.$ 7.8 Hz). ${ }^{13} \mathrm{C} \mathrm{NMR}\left(\mathrm{CDCl}_{3}\right): 189.3$, 165.9, 156.7, 151.3, 145.1, 144.0, 138.2, 137.1, 134.7, 133.9, 131.8, 130.7, 129.1, 128.8, 128.4, 128.0, 125.6, 115.2. MS: $m / z$ (\%) $413(\mathrm{M}+2,50), 412\left(\mathrm{M}^{+}\right.$1, 60), 386 (25), 105 (100). Anal. calcd. for $\mathrm{C}_{24} \mathrm{H}_{14} \mathrm{ClN}_{3} \mathrm{O}_{2}$ (411.8): C 69.99; H 3.43; N 10.20. Found: C 69.90; H 3.39; N 10.15.

\section{Acknowledgements}

The support of the University of Kuwait received through research grant \# Sc 10/07 and the facilities of ANALAB and SAF (grants no. GS01/01, GS01/03, GS03/01) are gratefully acknowledged.

\section{References}

1. Gabriel, S.; Colman, J. Chem. Ber. 1899, 32, 398.

2. Oates, H. F.; Stoker, L. M. Clin. Exp. Pharmacol. Physiol. 1981, 8, 133.

3. Maxwell, G. M.; Ness, D.; Rencis, V. Eur. J. Pharmacol. 1981, 69, 471.

4. Worms, P.; Gueudet, C. Biziere, K. Life Sci. 1986, 39, 2199.

5. Brooks, D. W.; Basha, A.; Kerdesky, F. A.; Holms, J. A.; Ratajcyk, J. D.; Bhatia, P.; Moore, J.; Martin, J. G.; Schmidt, S. ; Albert, D.; Dyer, R.; Yang, P.; Carter, G. W. Bioorg Med. Chem. Lett. 1992, 2, 1357.

6. Sayed, G. H.; Radwan, A.; Mohamed, A.; Shiba, S. A.; Khalil, M. Chin. J. Chem. 1992, 10, 475.

7. Butnariu, R. M.; Caprous, M. D.; Bejan, V.; Ungureanu, M.; Poiata, A.; Tuchilus, C.; Florescu, M.; Margareta, I. J. Heterocycl. Chem. 2007, 44, 1149.

8. Nomoto, Y.; Takai, H.; Ohno, T.; Nagashima, K.; Yao, K.; Yamada, K.; Kubo, K.; Ichimura, M.; Mihara, A.; Kase, H. J. Med. Chem. 1996, 39, 297.

9. Matsuda, T.; Aoki, T.; Koshi, T.; Ohkuchi, M.; Shigyo, H. Bioorg. Med. Chem. Lett. 2001, 11, 2369.

10. Barbaro, R.; Betti, L.; Botta, M.; Corelli, F.; Giannaccini, G.; Maccari, L.; Manetti, F.; Strapaghetti, G. Corsano, S. J. Med. Chem. 2001, 44, 2118.

11. Wermuth, C.; Schlewer, S.; Bourguignon, J.; Maghioros, G.; Bouchet, M.; Moire, C.; Kan, J.; Worms, P.; Biziere, K. J. Med. Chem. 1989, 32, 528.

12. Wermuth, C.; Bourguignon, J.; Schlewer, G.; Gies, J.; Schoenfelder, J.; Melikian, A.; 
Bouchet, M.; Chantureux, D.; Molimard, J.; Heaulme, M.; Chambon, J.; Biziere, K. J. Med. Chem. 1987, 30, 239.

13. Elnagdi, M. H.; Al-Awadi, N. A.; Abdelhamed, I. A. Adv. Heterocycl. Chem. 2009, 97, 1.

14. Al-Awadi, N. A.; Elnagdi, M. H.; Ibrhim, Y. A.; Kaul, K. Tetrahedron 2001, 57, 16.

15. Al-Awadi, N. A.; Ibrahim, M. R.; Abdelhamed, I. A.; Elnagdi, M. H. Tetrahedron 2008, 64, 8202.

16. Al-Matar, H. M.; Khalil, K. M.; Meier, M.; Kolshorn, H.; Elnagdi, M. H. Arkivoc 2008, (xvi), 288. (b) Ghozlan, S. A.; Abdelhamed, I. A.; Hassaneen, H. M.; Elnagdi, M. H. J. Heterocycl. Chem. 2007, 44, 105. (c) Al-Mousawi, S.; Moustafa, S.; Elnagdi, M. H. Heterocycles 2008, 75, 2201.

17. Reynolds, G. A.; Vanallan, J. A.; (Eds) Rabjohn N. In Org. Synth., Coll. Vol. IV, p 633. (b) Schmidt, B. Chem. Ber. 1901, 34, 2001.

18. Yoder, C. H.; Kennedy, S.; Snavely, F. H. J. Org. Chem. 1978, 43, 1077.

19. Dell'Erba. C.; Novi, M.; Petrillo, G.; Tavani, C. Tetrahedron 1993, 49, 235.

20. Crary, J. W.; Quayle, O. R.; Lester, C. T. J. Am. Chem. Soc. 1956, 78, 5584.

21. Kurt, B. Justus Liebigs Ann. Chem. 1959, 623, 109.

22. Hahn, W. E. Lodz. Towarz. Nauk., Wydziall III, 1960, 4, 101-15; Chem Abstr. 1961, 55 , 27941.

23. Hahn, W. E. Roczniki Chemii, 1959, 33, 65; Chem Abstr. 1959, 53, 89143. 